\title{
Scoliosis Research Society members attitudes towards physical therapy and physiotherapeutic scoliosis specific exercises for adolescent idiopathic scoliosis
}

\author{
Cindy L. Marti ${ }^{1}$, Steven D. Glassman ${ }^{2,3}$, Patrick T. Knott $^{4}$, Leah Y. Carreon ${ }^{3 *}$ and Michael T. Hresko ${ }^{5}$
}

\begin{abstract}
Background: Attitudes regarding non-operative treatment for adolescent idiopathic scoliosis (AIS) may be changing with the publication of BRAiST. Physiotherapeutic Scoliosis Specific Exercises (PSSE) are used to treat AIS, but high-quality evidence is limited. The purpose of this study is to assess the attitudes of members of the Scoliosis Research Society towards PSSE.

Methods: A survey was sent to all SRS members with questions on use of Physical Therapy (PT) and PSSE for AIS.

Results: The majority of the 263 respondents were from North America (175, 67 \%), followed by Asia (37, 14 \%) and Europe (36, $14 \%)$. The majority of respondents (166, $63 \%$ ) prescribed neither PT nor PSSE, 28 (11\%) prescribed both PT and PSSE, 39 (15\%) prescribe PT only and 30 (11 \%) prescribe PSSE only. PT was prescribed by 67 respondents, as an adjunct to bracing (39) and in small curves (32); with goals to improve aesthetics (27) and post-operative outcomes (25). Of the 196 who do not prescribe PT, the main reasons were lack of evidence (149) and the perception that PT had no value (112).

PSSE was prescribed by 58 respondents. The most common indication was as an adjunct to bracing (49) or small curves (41); with goals to improve aesthetics (36), prevent curve progression (35) and improve quality of life (31). Of the respondents who do not prescribe PSSE, the main reasons were lack of supporting research (149), a perception that PSSE had no value (108), and lack of access (63). Most respondents state that evidence of efficacy may increase the role of PSSE, with $85 \%$ (223 of 263) favoring funding PSSE studies by the SRS.

Conclusion: The results show that $22 \%$ of the respondents use PSSE for AIS, skepticism remains regarding the benefit of PSSE for AIS. Support for SRS funded research suggests belief that there is potential benefit from PSSE and the best way to assess that potential is through evidence development.
\end{abstract}

Keywords: Adolescent idiopathic scoliosis, Scoliosis specific exercise, Survey

\section{Background}

Physiotherapeutic Scoliosis Specific Exercise (PSSE) has been advocated as a potentially valuable tool in the treatment of adolescent idiopathic scoliosis (AIS) [1-11]. Postulated treatment effects include preventing curve progression, minimizing respiratory dysfunction, preventing spinal pain syndromes, and improving aesthetics

\footnotetext{
* Correspondence: leah.carreon@nortonhealthcare.org

${ }^{3}$ Norton Leatherman Spine Center, 210 East Gray Street, Suite 900, Louisville 40202, Kentucky, USA

Full list of author information is available at the end of the article
}

via postural correction [1-11]. These treatment effects may lead to avoidance of brace treatment or surgery $[12,13]$. PSSE is also commonly used in conjunction with brace treatment, and is suggested to increase the efficacy of bracing [14-16]. In general, the role of PSSE is more widely accepted in Europe versus North America.

The evidence-base supporting PSSE treatment regimen is very limited, as most of the published literature consists of case studies and small cohort studies. A systematic review in 2012 identified only 12 relevant studies and described the evidence as poor quality because all 
were at level IV or lower in terms of their Level of Evidence $[6,9]$. This deficiency is due in part to inconsistent indications for PSSE treatment, the diversity of therapy programs, ranging from outpatient postural training to intensive and prolonged in-patient regimens, and the difficulty in assuring compliance when patients are receiving therapy over an extended period. The best available literature, is a recent published randomized clinical trial of 110 patients that demonstrates PSSE to be superior to standard physical therapy (PT) in patients older than 10 years with small AIS curves $\left(10^{\circ}\right.$ to $\left.25^{\circ}\right)$ and Risser sign $<2$ [5].

Despite limited high quality evidence, the possibility of effective nonsurgical treatment for AIS is obviously attractive. Although U.S. surgeons have historically been skeptical regarding the role of these conservative exercise treatment options, attitudes may be changing. Publication of the BRAiST study, which demonstrated efficacy of bracing for AIS based upon clearly defined clinical parameters [17], has encouraged both patients and surgeons to the potential for brace treatment. At least in the public sector, this interest seems to extend beyond bracing to encompass a wider array of nonsurgical options.

The purpose of this study is to explore the attitudes of scoliosis surgeons regarding Physiotherapeutic Scoliosis Specific Exercise (PSSE). Beyond questions of awareness and acceptance, we sought to determine whether surgeons believe that evidence development regarding PSSE was an important research priority.

\section{Methods}

After receiving Institutional Board Approval, a webbased survey was sent to all 1200 Active and Candidate members of the Scoliosis Research Society. The survey (Table 1) included questions regarding membership status, training and years in practice. For the respondents who prescribe standard PT or PSSE, their indications and goals for standard PT and PSSE were queried. For those who do not prescribe standard PT or PSSE, they were further queried on why they do not prescribe standard PT or PSSE. In addition, respondents were asked if they have observed an increased interest in PSSE, what evidence of this they have observed, and if they anticipate an increased role for PSSE in the future. All analysis was performed using PASW Statistics GradPack 17.0 (Chicago, IL: SPSS Inc.). Fisher's exact test was used to determine difference in responses between regions of practice, years in training and type of training.

\section{Results}

There were a total of 266 respondents (22\%). Three were excluded from the analysis as they indicated that that they did not treat AIS patients, leaving 263 respondent surveys available for analysis. The majority of respondents $(175,67 \%)$ were from North America, with an almost equal number from Asia (37, 14 \%) and Europe (36, 14 \%), a small number of surgeons from South America and the Middle East.

Almost all the respondents were Orthopedic Surgeons; 159 (60 \%) categorized their spinal deformity training primarily as Orthopedic Spine and 95 (36\%) as Pediatric Orthopedics. Clinical experience, measured by years in practice, was almost equally represented, with most $(110,42 \%)$ in practice for more than 20 years, 81 (31\%) have been in practice between 11 and 20 years and 69 $(26 \%)$ have been in practice for ten years or less.

The majority of respondents (166, $63 \%)$ prescribed neither PT nor PSSE, 28 (11\%) prescribed both PT and PSSE, 39 (15\%) prescribe PT only and 30 (11\%) prescribe PSSE only. Sixty-seven (25\%) surgeons prescribe standard PT for their patients with AIS. Although the reported use of standard PT was more common in Europe, there was no statistically significant difference in prescribing pattern based on region, with an almost equal proportion of surgeons in Asia, South America and North America. There was no statistically significant difference in prescribing pattern based on years in practice, or training (Table 2).

Among the 67 surgeons who prescribe standard PT, the most common indication was the use of standard PT in conjunction with brace treatment (58\%), small curves (48 \%) or post-operatively (Table 3 ). Only $25 \%$ of surgeons prescribed PT for treatment of pain. However, alleviation of pain was chosen as the most common goal of therapy (72\%). Improving aesthetics (40\%) and improving post-operative outcomes $(37 \%)$ were the next most common goals of standard PT. Of the 196 (75 \%) surgeons who do not refer AIS patients for standard PT, the majority $(149,76 \%)$ stated the lack of supporting research as the most common reason, followed by the perception that standard PT had no value in the treatment of AIS (112, $57 \%$ ).

Fifty-eight (22\%) surgeons reported referring their AIS patients for PSSE. There was no statistically significant difference in referral pattern based on region or surgical training. Surgeons in practice for ten years or less were less likely to refer for PSSE compared to surgeons who have been in practice for a longer period of time (Table 4).

Among the 58 surgeons who refer patients for PSSE, the most common indication was similar to standard PT: in conjunction with brace treatment (84 \%) or for small curves (72 \%) (Table 5). However, unlike standard PT, PSSE was less often prescribed for treatment of pain (3\%). Consistent with the respondents indication for referral, stated goals for PSSE were most commonly to improve aesthetics $(62 \%)$, to prevent curve progression (60\%) and to improve quality of life (53\%). The most common specific PSSE used was Schroth (33, $57 \%$ ), 
Table 1 Survey questions and responses

\begin{tabular}{ll}
\hline Question & Response \\
Member type & Active \\
& Candidate
\end{tabular}

\section{Country}

Birth date

Please describe your training.

Candidate

How many years have you been in practice?

Do you treat AIS in your practice?

Do you refer AIS patients for STANDARD physical therapy?

Which patients? (check all that apply)

Indicate primary goals of referring for STANDARD physical therapy? (check all that apply)

Why don't you refer? (check all that apply)

Do you refer patients for SCOLIOSIS SPECIFIC EXERCISE?

Which methods? (check all that apply):

Which patients? (check all that apply)

What are your goals for SCOLIOSIS SPECIFIC EXERCISE (SSE)? (check all that apply)

Why don't you refer? (check all that apply)

Have you observed an increased interest in SCOLIOSIS SPECIFIC EXERCISE?

In what ways do you see evidence of this change? (check all that apply)

Why not? (check all that apply)

Do you anticipate an increased role for SSE in the future?

What would facilitate this change?

Do you support the use of SRS research funds to support higher quality research regarding the potential benefit of SSE for AIS? YeS/No
Orthopedic Spine Surgeon

Pediatric Orthopedic Surgeon

Neurosurgical Spine Surgeon

Other (please specify)

0-10 years

11-20 years

$>21$ years

Yes/No

Yes/No

Small curves - not braced

Curves in braces

Pre-surgical

Post-surgical

Only for families who request therapy

Patients with symptoms/pain

Prevent curve progression

Improve aesthetics via postural correction

Prevent or treat spinal pain

Prevent or treat respirary dysfunction

Prevent surgery

Improve post-surgical outcome with pre-op PT

Postpone surgery

Lack of perceived value in standard PT

Lack of access

Lack of patient interest/compliance

Lack of perceived value in standard PT

Lack of research to support standard PT

Cost to patient

Yes/No

Schroth

Lyon Method

Side Shift Method

Functional Individual Therapy of Scoliosis

Scientific Exercise Approach to Scoliosis

Small curves - not braced

Curves in braces

Improved posture/Aesthetic

Post-surgical

Pre-surgical

Only for families who ask

Improved posture/Aesthetic

Improving surgical outcome

Postponing surgery

Preventing Curve Progression

Preventing surgery

Improved posture/Aesthetic

Lack of access to SSE trained therapists

Lack of research to support SSE

Lack of perceived value in SSE

Lack of access to SSE trained therapists

Lack of patient interest/compliance

Yes/No

Hospitals and Clinics in your area are promoting SSE

More research/publications about PT or SSE

Patients and families are asking for PT or SSE

Doctors are referring more frequently to PT or SSE

Hospitals and Clinics in your area are promoting SSE

Lack of patients/family interest in SSE

Lack of physician education or interest about benefit of SSE

Lack of research/publications about of SSE

Hospitals and Clinics in your area do not provide SSE Yes/No

Please add any additional perspectives you would like to share: 
followed by Side Shift (13, $22 \%)$, SEAS (12, $21 \%)$ and FITS (11, $19 \%$ ) with almost similar distribution (Table 5). For the 204 (78 \%) of surgeons who do not refer for PSSE, the main reasons were similar to standard PT, the lack of supporting research (149, $73 \%$ ), followed by the perception that PSSE had no value (108, $53 \%)$. In addition, 63 (31\%) reported that they had no access to a facility providing PSSE.

Slightly more than half of the surgeons (140, $53 \%$ ) noted an increased interest in PSSE, based mostly from inquiries from patients and their families (Table 6). When asked what factors might increase the role of PSSE in the treatment of AIS, most surgeons stated increased evidence of efficacy. Perhaps, due to this identified need, a large majority of surgeons $(223,86 \%)$ favored funding PSSE studies by the SRS.

\section{Discussion}

Treatment effectiveness of nonsurgical interventions for AIS remains under studied [18-20], with contradictory recommendations from research studies $[2,8$, 20] and insufficient evidence to guide treatment. Specifically, Physiotherapeutic Scoliosis Specific Exercise (PSSE) treatment for AIS, although increasingly prevalent in Europe [1], have not been considered effective in North America. Spinal deformity surgeons, the Scoliosis Research Society and the Society on Scoliosis Orthopaedic and Rehabilitation and Treatment (SOSORT)

Table 2 Respondent characteristics in prescribing standard physical therapy

\begin{tabular}{|c|c|c|c|}
\hline & \multicolumn{2}{|c|}{ Standard physical therapy } & \multirow[t]{2}{*}{ p-value } \\
\hline & $\overline{\mathrm{No}}$ & Yes & \\
\hline Total & 196 & 67 & \\
\hline Region & & & 0.591 \\
\hline North America & 132 & 43 & \\
\hline Asia & 29 & 8 & \\
\hline South America & 7 & 2 & \\
\hline Europe & 23 & 13 & \\
\hline Middle East & 5 & 1 & \\
\hline Years in Training & & & 0.272 \\
\hline 0 to 10 & 54 & 15 & \\
\hline 11 to 20 & 55 & 26 & \\
\hline 21 or more & 85 & 26 & \\
\hline Training & & & 0.200 \\
\hline Orthopedic Spine Surgeon & 113 & 46 & \\
\hline Pediatric Orthopedic Surgeon & 74 & 21 & \\
\hline Neurosurgical Spine Surgeon & 2 & 0 & \\
\hline Other & 7 & 0 & \\
\hline
\end{tabular}

Table 3 Stated indications and goals for standard physical therapy $(N=67)$

\begin{tabular}{lll}
\hline Indication & Frequency & Percentage \\
\hline Small curves & 32 & $48 \%$ \\
Braced Curves & 39 & $58 \%$ \\
Pre-operative & 17 & $25 \%$ \\
Post-operative & 25 & $37 \%$ \\
Patient request & 7 & $10 \%$ \\
Pain & 17 & $25 \%$ \\
Goals & & \\
Prevent Progression & 12 & $18 \%$ \\
Improve Aesthetics & 27 & $40 \%$ \\
Treat Pain & 48 & $72 \%$ \\
Treat Respiratory Symptoms & 11 & $16 \%$ \\
Prevent Surgery & 6 & $9 \%$ \\
Postpone Surgery & 3 & $4 \%$ \\
Improve Post operative Outcomes & 25 & $37 \%$ \\
Maintain Improve Core Strength & 7 & $10 \%$ \\
Exercise with Bracing & 1 & $1 \%$ \\
Improved quality of life & 1 & $1 \%$ \\
Maintain flexibility & 1 & $1 \% \%$ \\
To get back to activities & 1 & $1 \% \%$ \\
Why don't you prescribe Standard & & \\
Physical Therapy? & $14 \%$ \\
No access & & \\
No Patient Interest & & \\
No value & & \\
No research & & \\
\hline
\end{tabular}

[20] have recognized the lack of evidence supporting exercise treatment. The SRS funded a pilot study of PSSE, which resulted in a current RCT ongoing in Canada comparing standard PT to PSSE in adolescent scoliosis patients at a single center [21]. Despite a steady increase in the discussion surrounding PSSE, the view of PSSE among spinal deformity surgeons remains largely unknown. This study reports on survey results from 263 spinal deformity surgeons, relating to their opinions and attitudes concerning exercise treatment for Adolescent Idiopathic Scoliosis (AIS).

Reflective of the lack of strong evidence to support PT or PSSE to treat AIS, the majority (63\%) of respondents prescribed neither. Of those prescribing PT or PSSE, the number prescribing either or both was similar. In addition, despite the impression that PT and PSSE are more widely used in Europe [7-9, 15, $19,20,22]$ compared to North America, our study showed that there was no difference in prescribing patterns among the respondents based on region. 
Table 4 Respondent characteristics in prescribing Scoliosis specific exercises

\begin{tabular}{|c|c|c|c|}
\hline & \multicolumn{2}{|c|}{ Scoliosis specific exercises } & \multirow[t]{2}{*}{$p$-value } \\
\hline & No & Yes & \\
\hline Total & 204 & 58 & \\
\hline Region & & & 0.520 \\
\hline North America & 140 & 34 & \\
\hline Asia & 25 & 12 & \\
\hline South America & 7 & 2 & \\
\hline Europe & 27 & 9 & \\
\hline Middle East & 5 & 1 & \\
\hline Years in Training & & & 0.022 \\
\hline 0 to 10 & 62 & 7 & \\
\hline 11 to 20 & 60 & 21 & \\
\hline 21 or more & 81 & 29 & \\
\hline Training & & & 0.824 \\
\hline Orthopedic Spine Surgeon & 124 & 35 & \\
\hline Pediatric Orthopedic Surgeon & 72 & 22 & \\
\hline Neurosurgical Spine Surgeon & 2 & 0 & \\
\hline Other & 6 & 1 & \\
\hline
\end{tabular}

Although, there was no difference in prescribing pattern for standard PT based on the surgeon's years in practice, older surgeons were more likely to prescribe PSSE. PSSEs were developed in an era where clinical traditions and methods were passed on [23], unlike today where knowledge is spread through rigorous study design and publication of findings. Thus, surgeons who have been in practice longer may have had more exposure to PSSEs.

Consistent with current clinical indications, the majority of the respondents prescribe PT and PSSE for small curves $[7,20,22]$ and as an adjunct to brace treatment [14-16]. Current guidelines recommend PSSE in curves less than $25^{\circ}$ to avoid bracing by stabilizing the spine, and obtaining 3D autocorrection of the spine, pelvis and rib cage [20]. Twenty-five degrees is the generally accepted threshold at which brace treatment is recommended [20, 24] Although $25 \%$ of surgeons prescribed PT for treatment of pain; alleviation of pain was chosen as the most common goal of therapy, and only $3 \%$ prescribed PSSE for pain. This may illustrate some confusion regarding the benefits of exercise in the AIS population.

Despite an increased patient awareness of nonsurgical treatments for AIS, the majority of surgeons do not prescribe PT or PSSE. Respondents stated that they did not refer AIS patients for standard PT or PSSE as they felt that these treatments were of no value in AIS and that there was no supporting research. Recognizing this
Table 5 Stated type, indications and goals of Scoliosis specific exercise $(N=58)$

\begin{tabular}{|c|c|c|}
\hline Scoliosis specific exercise & Frequency & Percentage \\
\hline Schroth & 33 & $57 \%$ \\
\hline SEAS & 12 & $21 \%$ \\
\hline FITS & 11 & $19 \%$ \\
\hline Side Shift & 13 & $22 \%$ \\
\hline Lyon & 4 & $7 \%$ \\
\hline Dobomed & 1 & $2 \%$ \\
\hline \multicolumn{3}{|l|}{ Indications } \\
\hline Small curves & 42 & $72 \%$ \\
\hline Braced Curves & 49 & $84 \%$ \\
\hline Pre-operative & 18 & $31 \%$ \\
\hline Post-operative & 12 & $21 \%$ \\
\hline Patient request & 3 & $5 \%$ \\
\hline Pain & 2 & $3 \%$ \\
\hline Non-athletes in particular & 1 & $2 \%$ \\
\hline \multicolumn{3}{|l|}{ Goals } \\
\hline Prevent Progression & 35 & $60 \%$ \\
\hline Prevent Surgery & 19 & $33 \%$ \\
\hline Postpone Surgery & 12 & $21 \%$ \\
\hline Improve Post-operative Outcomes & 20 & $34 \%$ \\
\hline Improve Aesthetics & 36 & $62 \%$ \\
\hline Improve quality of life & 31 & $53 \%$ \\
\hline Decrease pain when present & 1 & $2 \%$ \\
\hline General Fitness & 1 & $2 \%$ \\
\hline Improve brace results & 1 & $2 \%$ \\
\hline Muscle strengthening & 2 & $3 \%$ \\
\hline \multicolumn{3}{|c|}{$\begin{array}{l}\text { Why don't you prescribe Scoliosis Specific } \\
\text { Exercise }\end{array}$} \\
\hline No access & 63 & $31 \%$ \\
\hline No Patient Interest & 12 & $6 \%$ \\
\hline No value & 108 & $53 \%$ \\
\hline No research & 149 & $73 \%$ \\
\hline Cost & 4 & $2 \%$ \\
\hline Lack of exposure/experience & 5 & $2 \%$ \\
\hline
\end{tabular}

need, there was an overwhelming response that there was a need for better evidence demonstrating efficacy and most favored funding PSSE research using SRS research dollars.

There are limitations to this study, foremost of which is the relatively small number of respondents at $22 \%$. This may raise the possibility of selection bias in that only surgeons with a specific interest in the non-operative treatment of AIS may have responded. Yet a substantial number of respondents indicated that they do not prescribe either 
Table 6 Responses to "Have you observed an increased interest in SSE?"

\begin{tabular}{ll}
\hline Yes & 140 \\
\hline Patients ask & 113 \\
More referrals & 18 \\
More research/publications & 39 \\
Hospitals promoting & 20 \\
No & 123 \\
No patient interest & 49 \\
No physician education & 50 \\
No research & 84 \\
Not offered & 51 \\
\hline
\end{tabular}

PT or PSSE. Still, this is a starting point in initiating the discussion on the need for a more robust study on the effectiveness of either PT or PSSE in the treatment of AIS.

While effective nonsurgical treatment for AIS would represent an important benefit for many patients, demonstration of treatment efficacy has generally proven difficult. However, the success of the BRAiST study has created a renewed focus on the potential for other nonsurgical treatments, and an increased optimism that nonsurgical treatments may be effectively studied. The results of this survey demonstrate significant skepticism regarding the benefit of either standard PT or PSSE in the management of AIS, which is to be expected given the lack of evidence. On the other hand, the fact those spinal deformity surgeons indicated overwhelming support for SRS funded research suggests a belief that at least some patients might benefit from PSSE treatment. It certainly indicates a growing understanding among SRS surgeons that evidence development is the optimal way to answer these important questions.

The results of this survey show that $22 \%$ of the respondents use PSSE for AIS, although skepticism remains regarding the benefit of PSSE for AIS. Support for SRS funded research suggests belief that there is potential benefit from PSSE and the best way to assess that potential is through evidence development.

\section{Competing interests}

CLM, PTK has nothing to disclose. SDG is an employee of and receives research grants from Norton Healthcare; and receives royalties from Medtronic. LYC is on the editorial advisory board of Spine and The Spine Journal, is a member of Institutional Review Board of the University of Louisville and is a Board Member of the Research Committee of the Socliosis Research Society and the Global Evidence Advisory Board of Medtronic; is an employee of and receives research grants from Norton Healthcare; receives research grants from AO Spine, Scoliosis Research Society and the Orthopedic Research and Educational Fund; received funds for travel from the Orthopedic Research and Educational Fund, Institutional Review Board of the University of Louisville, Association for Collaborative Spine Research and the Center for Spine Surgery and Research, Region of Southern Denmark, received honoraria from the National institutes of Health, Medtronic and the Children's Tumor Foundation. MTH received payments for lectures from DePuy Spine and manuscript preparation from the New England Journal of Medicine. SDG, LYC and MTH: Nuvasive provided funds directly to database company. No funds were paid directly to Individual or Individual's Institution 06/2012-04/2015

\section{Authors' contributions}

CLM: conception and design, drafting the article, critically revising the article, reviewed submitted version of manuscript. SDG: acquisition of data, analysis and interpretation of data, drafting the article, critically revising the article, reviewed submitted version of manuscript, administrative/technical/material support. PTK: critically revising the article, reviewed submitted version of manuscript. LYC: critically revising the article, reviewed submitted version of manuscript, approved the final version of the manuscript on behalf of all authors, statistical analysis. MTH: critically revising the article, reviewed submitted version of manuscript. All authors read and approved the final manuscript.

\section{Author details}

'Spinal Dynamics of Wisconsin, 2300 North Mayfair Road, Suite 555, Wauwatosa, WI 53226, USA. ²Department of Orthopaedic Surgery, University of Louisville School of Medicine, 550 S. Jackson St., 1st Floor ACB, Louisville 40202, Kentucky, USA. ${ }^{3}$ Norton Leatherman Spine Center, 210 East Gray Street, Suite 900, Louisville 40202, Kentucky, USA. ${ }^{4}$ Rosalind Franklin University of Medicine and Science, 3333 Green Bay Road, North Chicago, IL 60064, USA. ${ }^{5}$ Department of Orthopaedic Surgery, Children Hospital Boston, Harvard Medical School, Boston, MA 02115, USA.

Received: 17 March 2015 Accepted: 19 May 2015

Published online: 27 May 2015

\section{References}

1. Bettany-Saltikov J, Parent E, Romano M, Villagrasa M, Negrini S. Physiotherapeutic scoliosis-specific exercises for adolescents with idiopathic scoliosis. Eur J Phys Rehabil Med. 2014;50(1):111-21.

2. den Boer WA, Anderson PG, Limbeek J, Kooijman MA. Treatment of idiopathic scoliosis with side-shift therapy: an initial comparison with a brace treatment historical cohort. Eur Spine J. 1999;8(5):406-10.

3. Dobosiewicz K, Durmala J, Kotwicki T. Dobosiewicz method physiotherapy for idiopathic scoliosis. Stud Health Technol Inform. 2008;135:228-36.

4. Fusco C, Zaina F, Atanasio S, Romano M, Negrini A, Negrini S. Physical exercises in the treatment of adolescent idiopathic scoliosis: an updated systematic review. Physiother Theory Pract. 2011;27(1):80-114.

5. Monticone M, Ambrosini E, Cazzaniga D, Rocca B, Ferrante S. Active selfcorrection and task-oriented exercises reduce spinal deformity and improve quality of life in subjects with mild adolescent idiopathic scoliosis. Results of a randomised controlled trial. Eur Spine J. 2014;23(6):1204-14.

6. Mordecai SC, Dabke HV. Efficacy of exercise therapy for the treatment of adolescent idiopathic scoliosis: a review of the literature. Eur Spine J. 2012;21(3):382-9.

7. Negrini S, Aulisa AG, Aulisa L, Circo AB, de Mauroy JC, Durmala J, et al. 2011 SOSORT guidelines: Orthopaedic and Rehabilitation treatment of idiopathic scoliosis during growth. Scoliosis. 2012;7(1):3

8. Negrini S, Aulisa L, Ferraro C, Fraschini P, Masiero S, Simonazzi P, et al. Italian guidelines on rehabilitation treatment of adolescents with scoliosis or other spinal deformities. Eura Medicophys. 2005;41(2):183-201.

9. Romano M, Minozzi S, Bettany-Saltikov J, Zaina F, Chockalingam N, Kotwicki T, Maier-Hennes A, Negrini S. Exercises for scoliosis in teens http://summaries.cochrane.org/CD007837/BACK_exercises-for-scoliosis-inteens\#sthash.jlk3B6ui.dpuf. Published Online: 17 October 2012 accessed October 3, 2014

10. Weiss HR, Weiss G, Petermann F. Incidence of curvature progression in idiopathic scoliosis patients treated with scoliosis in-patient rehabilitation(SIR): an age- and sex-matched controlled study. Pediatr Rehabil. 2003;6(1):23-30.

11. Weiss HR, Weiss G. Curvature progression in patients treated with scoliosis in-patient rehabilitation-a sex and age matched controlled study. Stud Health Technol Inform. 2002;91:352-6.

12. Negrini S, Fusco C, Minozzi S, Atanasio S, Zaina F, Romano M. Exercises reduce the progression rate of adolescent idiopathic scoliosis: results of a comprehensive systematic review of the literature. Disabil Rehabil. 2008;30(10):772-85.

13. Negrini S, Zaina F, Romano M, Negrini A, Parzini S. Specific exercises reduce brace prescription in adolescent idiopathic scoliosis: a prospective controlled cohort study with worst-case analysis. J Rehabil Med. 2008;40(6):451-5. 
14. Carman D, Roach JW, Speck G, Wenger DR, Herring JA. Role of exercises in the Milwaukee brace treatment of scoliosis. J Pediatr Orthop. 1985;5(1):65-8.

15. Rivett $L$, Stewart A, Potterton J. The effect of compliance to a Rigo System Cheneau brace and a specific exercise programme on idiopathic scoliosis curvature: a comparative study: SOSORT 2014 award winner. Scoliosis. 2014;9:5.

16. Zaina F, Negrini S, Atanasio S, Fusco C, Romano M, Negrini A. Specific exercises performed in the period of brace weaning can avoid loss of correction in Adolescent Idiopathic Scoliosis (AIS) patients: Winner of SOSORT's 2008 Award for Best Clinical Paper. Scoliosis. 2009;4:8.

17. Weinstein $S L$, Dolan $L A$, Wright JG, Dobbs MB. Effects of bracing in adolescents with idiopathic scoliosis. N Engl J Med. 2013;369(16):1512-21.

18. Moen KY, Nachemson AL. Treatment of scoliosis. An historical perspective. Spine (Phila Pa 1976). 1999;24(24):2570-5.

19. Płaszewski M, Bettany-Saltikov J. Non-surgical interventions for adolescents with idiopathic scoliosis: an overview of systematic reviews. PLoS One. 2014;9(10):e110254. d.

20. Weiss HR, Negrini S, Hawes MC, Rigo M, Kotwicki T, Grivas TB, et al. Physical exercises in the treatment of idiopathic scoliosis at risk of brace treatment SOSORT consensus paper 2005. Scoliosis. 2006;1:6.

21. Parent EC, Fortin C. A Multicenter Randomized Controlled Trial to Compare the Effect of Schroth Exercises to Standard Care on Curve Characteristics, Posture, and Quality Of Life in Adolescents With Idiopathic Scoliosis. (MultiSETS) https:// clinicaltrials.gov/ct2/show/NCT01610908?term=scoliosis+exercise\&rank=1 Published online April 2, 2012 accessed November 15, 2014

22. Weiss HR. Spinal deformities rehabilitation - state of the art review. Scoliosis. 2010:5:28

23. Weiss HR. The method of Katharina Schroth - history, principles and current development. Scoliosis. 2011;6:17.

24. Lonstein JE. Scoliosis: surgical versus nonsurgical treatment. Clin Orthop Relat Res. 2006:443:248-59. Review. PubMed.

\section{Submit your next manuscript to BioMed Central and take full advantage of:}

- Convenient online submission

- Thorough peer review

- No space constraints or color figure charges

- Immediate publication on acceptance

- Inclusion in PubMed, CAS, Scopus and Google Scholar

- Research which is freely available for redistribution 